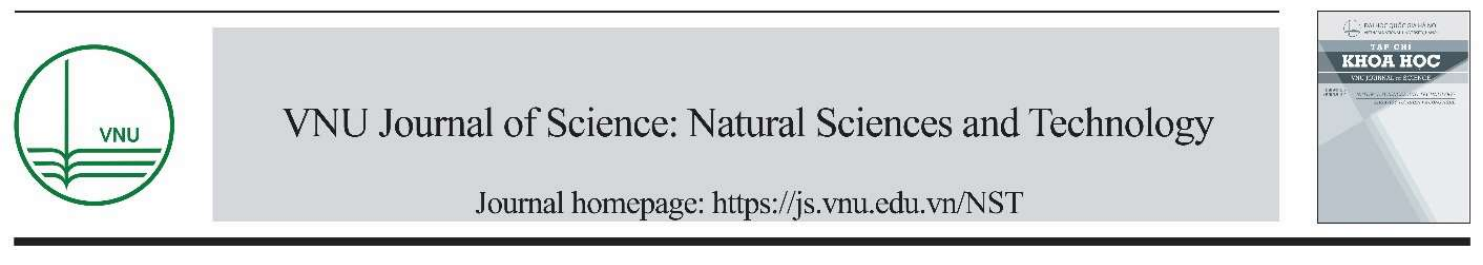
Original Article

\title{
Taxillus Thibetensis (Lecomte) Danser (Loranthaceae) a New Record Species For Flora of Vietnam
}

\author{
Chi Toan Le ${ }^{1}$, Van Du Nguyen ${ }^{2}$, Thu Lan Pham ${ }^{1}$, Thi Ngat Le ${ }^{3}$, Bing Liu ${ }^{4}$ \\ ${ }^{1}$ Hanoi Pedagogical University No. 2, 32 Nguyen Van Linh, Xuanhoa, Phucyen, Vinhphuc, Vietnam \\ ${ }^{2}$ Vietnam Academy of Science and Technology, 18 Hoang Quoc Viet, Cau Giay, Hanoi, Vietnam \\ ${ }^{3}$ Hung Vuong University, Nong Trang, Viet Tri, Phu Tho, Vietnam \\ ${ }^{4}$ Institute of Botany, Chinese Academy of Sciences, Beijing 100093, China
}

Received 11 September 2020

Accepted 5 May 2021

\begin{abstract}
Taxillus thibetensis (Lecomte) Danser (Loranthaceae), a species previously known only from China, is newly recorded from Vietnam. A specimen discovered in the rainforest of Lao Cai province, Northern Vietnam was identified as Taxillus thibetensis based on both morphological and molecular data. The Vietnamese individuals of Taxillus thibetensis are closely related to $T$. thibetensis in China. A detailed description, illustration and data on distribution, ecology, phenology of Vietnamese Taxillus thibetensis are provided.
\end{abstract}

Keywords: Loranthaceae, Taxillus Thibetensis, New Record, Morphology, Molecular Data.

\section{Introduction}

Taxillus Tiegh., the genus of subtribe Scurrulinae (Loranthaceae) includes ca. 35 species distributed from China to Southeast Asia and one species in Africa (coastal area of Kenya) [1-3]. Pham [4] recorded four species of Taxillus in Vietnam including: T. balansae, T. delavayi, $T$. kwangtungensis, T. chinensis. However, Nguyen [5] lumped members of Taxillus and Scurrula L. into the genus Taxillus, thus this genus includes

\footnotetext{
*Corresponsding author.

Email address: Lechitoan@hpu2.edu.vn.

https://doi.org/10.25073/2588-1140/vnunst.5126
}

13 species in Vietnam. Le et al. [6] suggested to recognized Scurrula and Taxillus as two separate genera, and the genus Taxillus includes 5 species in Vietnam.

During the field trip in October 2019, the Taxillus specimens were collected in Ban Khoang commune, Sapa district, Lao Cai province of Vietnam. After analyzed the morphological characters of the specimens, we 
recognized the plant is Taxillus thibetensis (Lecomte) Danser, a species found only in China before. This is the first time T. thibetensis found in Vietnam and this newly recorded species updates the total number of Taxillus in Vietnam to six species. In this article, the identifying characteristics, pictures, and phylogenetic position, of Taxillus thibetensis are presented and discussed.

\section{Materials and Methods}

\subsection{Taxon Sampling, DNA Extraction, Amplification, Sequencing}

Studied specimens include dry specimens keept in the herbarium at Institute of Ecology and Biological Resource $(\mathrm{HN})$, and department of Biology, Hanoi Pedagogical University No. 2, together with new specimens obtained during the survey in Lao Cai Province. Furthermore, the morphology of Taxillus thibetensis was also compared to other specimens in herbaria: PE (Beijing, China), KUN (Kunming, China). The herbarium codes follow the Index Herbariorum (http://sweetgum.nybg.org/ih/).
The descriptions from the Flora of China account [1] has been assessed and used as the basis for an expanded description of the species. Nomenclatural practice follows the International Code of Nomenclature for algae, fungi, and plants (ICN) [7].

For the molecular analyses, we assembled all sequences of Taxillus and Scurrula for five genes from Genbank (NCBI) including nuclear smallsubunit ribosomal DNA (SSU rDNA), largesubunit ribosomal DNA (LSU rDNA), and three chloroplast DNA regions ( $r b c L, m a t K$ and $t r n L-$ F) (Table 1); the species Dendrophthoe longituba was selected as outgroup [3, 8-11]. Voucher information and GenBank accession numbers are listed in (Table S1). Moreover, we extracted genomic DNA and sequencing five gene regions of Taxillus thibetensis collected in Lao Cai, then added sequences of the Taxillus thibetensis to the datasets obtained from Genbank to construct the molecular phylogenetic trees. Genomic DNA was extracted from silica gel dried tissues following Doyle and Doyle [12].

Table 1. Primers used for PCR and sequencing in this study

\begin{tabular}{|l|l|l|l|}
\hline Locus & Primer & Sequence 5'-3' & Reference \\
\hline Chloroplast & & & \\
\hline$m a t K$ & $78 \mathrm{~F}$ & CAGGAGTATATTTATGCACT & Vidal-Russell \& Nickrent $[8,13]$ \\
\hline & $1420 \mathrm{R}$ & TCGAAGTATATACTTTATTCG & \\
\hline$r b c L$ & $1 \mathrm{~F}$ & ATGTCACCACAAACAGARAC & Vidal-Russell \& Nickrent $[8,13]$ \\
\hline & $889 \mathrm{R}$ & CTATCAATAACTGCATGCAT & \\
\hline trnL-F & $\mathrm{C}$ & CGAAATCGGTAGACGCTACG & Taberlet et al. [14] \\
\hline & $\mathrm{F}$ & ATTTGAACTGGTGACACGAG & \\
\hline Nuclear & & & \\
\hline LSUr DNA & $27 \mathrm{~F}$ & CCCGCTGAGTTTAAGCATA & Vidal-Russell \& Nickrent $[8,13]$ \\
\hline & $950 \mathrm{~F}$ & GCTATCCTGAGGGAAACTTC & \\
\hline SSUr DNA & $12 \mathrm{~F}$ & TCCTGCCAGTASTCATATGC & Vidal-Russell \& Nickrent $[8,13]$ \\
\hline & $1796 \mathrm{R}$ & CACCTACGGAAACCTTGTT & \\
\hline
\end{tabular}

Polymerase chain reactions and sequencing were performed using the primers designed by Vidal-Russell \& Nickrent $[8,13]$ and Taberlet et al. [14]. The primers used for conducting PCR and sequencing were presented in Table 1 . The PCR amplification reactions used MasterMix of the BioMed company. The PCR program consisted of $5 \mathrm{~min}$ at $95^{\circ} \mathrm{C}, 36$ cycles of $30 \mathrm{~s}$ at $95^{\circ} \mathrm{C}, 50 \mathrm{~s}$ at $49^{\circ} \mathrm{C}$, and $1 \mathrm{~min} 30 \mathrm{~s}$ at $72^{\circ} \mathrm{C}$, with a final extension of $10 \mathrm{~min}$ at $72^{\circ} \mathrm{C}$.

PCR products were purified on $1.0 \%$ agarose gels. The all PCR products were purified using 
BioMed multifunctional DNA fragment purification recovery kits then were sequenced using the amplification primers. The bidirectional sequencing was completed using the ABI 3730 DNA Sequencer (Applied Biosystems, Carlsbad, California, USA). The sequences were aligned in Geneious v.8.0.5 [15].

\subsection{Phylogenetic Analyses}

Both the maximum likelihood (ML) and Bayesian inference (BI) were carried out for the phylogenetic analyses.

The ML analysis was performed using the program RAxML 8.2.10 [16,17] with the GTR + I $+G$ substitution model for each molecular marker and the combined dataset at the Cyper Infrastructure for Phylogenetic Research (CIPRES; www.phylo.org). ML bootstrap analysis was implemented with 1000 replicates. Bayesian inference was conducted in MrBayses 3.1.2 [18]. The best-fitting models for each marker and the combined data set were determined by the Akaike information Criterion (AIC) as implemented in jModelTest 2.1.6 [19]. Bayesian analysis of the combined data set used the GTR + I + G model as determined in jModelTest. The MCMC algorithm was run for 5,000,000 generations with four Markov chain Monte Carlo (MCMC) and trees were sampled every 1000 generations. The program Tracer 1.6 [20] was used to check that effective sample size (ESS) for all relevant parameters were well above 200 indicating that stationarity probably had been reached. With the first $25 \%$ of sampled generations (2500 trees) discarded as burn-in, a $50 \%$ majorily-rule consensus tree and posterior probabilities (PP) were obtained using the remaining trees.

\section{Results and discussion}

Our molecular analyses based on combined dataset from five makers strongly supported the new samples collected from Lao Cai provice grouped with Taxillus thibetensis (the samples from China) with strong bootstrap support (BS:
100\%, PP: 1.0) (Figure 1). Furthermore, by comparing specimens of Taxillus, especial type specimen of Taxillus thibetensis in the herbaria and the description in the Flora of China, we identified the collection as $T$. thibetensis, a species previously unrecorded from Vietnam.

The results from molecular analyses also suggest to recognize two separate genera Taxillus and Scurrula with strongly supported. The Vietnamese individuals of Taxillus thibetensis are closely related to T. thibetensis in China (Figure 1). T. thibetensis were characteristic by abaxial surface tomentose while adaxial surface rapidly glabrescent, corolla exterior pilose with dense verticillate hairs and tip of bud ellipsoid (Figure 2).

\section{Taxonomic treatment}

Describe a new record species for flora of Vietnam:

Taxillus thibetensis (Lecomte) Danser, Bull. Jard. Bot. Buitenzorg, sér. 3, 10: 355. 1929.

Type:-CHINA: Dêqên County, Tsekou, 15 June 1895, R. P. Soulié 1340 (Syntype!, P); Yunnan Prov., Dêqên County, Tsekou, Monbeig s. n. (Syntype, P).

Description: Aerial parasite, shrubs 0.8-1.2 $\mathrm{m}$ tall, young stems mostly tomentose, becoming glabrous when older, hairs yellowish brown, rarely white, both verticillate and stellate. Young branchlets tomentose with dense rusty red scales. Branches black or gray, almost glabrous, subsmooth, scattered lenticellate. Leaves opposite or subopposite; young leaf with dense rusty red scales, petiole $1 \mathrm{~cm}$, pilose; leaf blade ovate or ovate-oblong, 6-11 $\times 3-5.5 \mathrm{~cm}$, leathery, abaxial persistently tomentose, adaxial rapidly glabrescent, lateral veins 5-8 pairs, base subrounded, margin entire or undulate, apex obtuse or acute. Inflorescences axillary or at leafless node, umbels 2-3-fascicled, 3-5flowered; peduncle and rachis $3-8 \mathrm{~mm}$. Flowers bisexual, 4-merous, zygomorphic, yellow-brown or brown, rarely white, tomentose; bracts ovate, ca. $1 \mathrm{~mm}$, apex acute. 


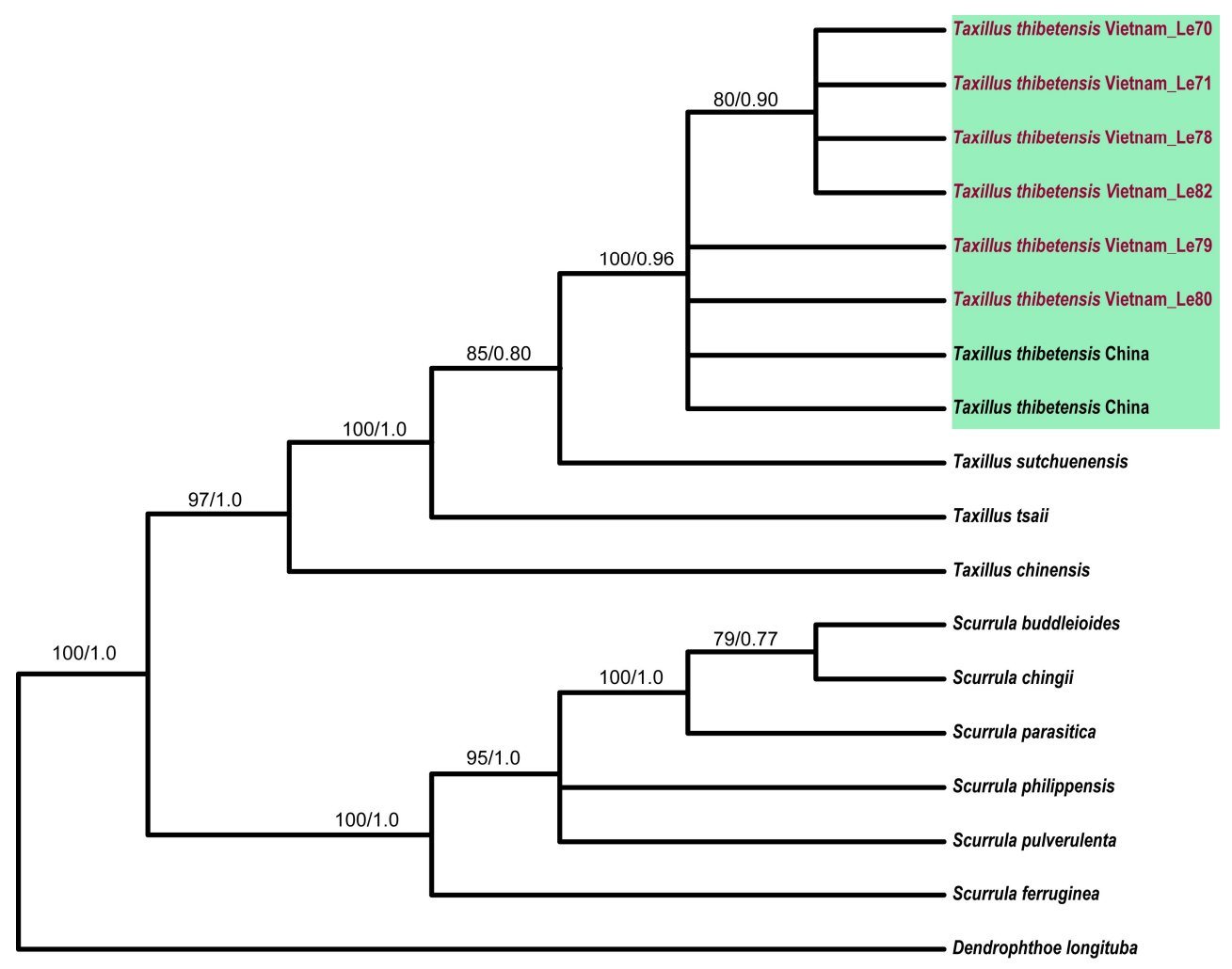

Figure 1. Majority rule consensus tree from a Bayesian analysis of the concatenated data set that includes the five genes. ML bootstrap values and posterior probabilities (PP) of the BI analysis are presented above the branches.

Pedicel 2-5 mm. Calyx ellipsoid, ca. $2 \mathrm{~mm}$, limb annular, entire or minutely 4-toothed. Mature bud tubular $2.2-3.2 \mathrm{~cm}$, tip ellipsoid. Corolla red, tube slightly curved, pilose with dense verticillate hairs, basal part inflated, lobes 4 , lanceolate, $7-8 \mathrm{~mm}$, split along one side at anthesis, reflexed. Stamens inserted at base of corolla lobes; filaments short, $1.5-2 \mathrm{~mm}$; anthers 3.5-4 mm, multilocellate. Ovary 1-loculed; placentation basal. Style filiform, stigma capitate. Berry yellowish, ovoid or ellipsoid, 5-10 × 4-6 $\mathrm{mm}$, granulose, pilose, with hairs, base tapering into stalk.

Habitat: Forests, mountain slopes, valleys, orchards, gardens; 2000-3000 m.
Phenology: Flowering in May-October; fruiting in August-November.

Distribution: New distributed points found in Vietnam are Lao Cai province. China

(Guizhou, SW Sichuan, SE Xizang, Yunnan).

Note: recorded hosts of Taxillus thibetensis including Quercus spp., Prunus spp. Kiu \& Gilbert [1] suggested that Castanea mollissima, Diospyros kaki, Pyrus pyrifolia, and Salix spp. also recorded as hosts of Taxillus thibetensis in China.

IUCN Red List category: There have been no comprehensive field surveys of populations 

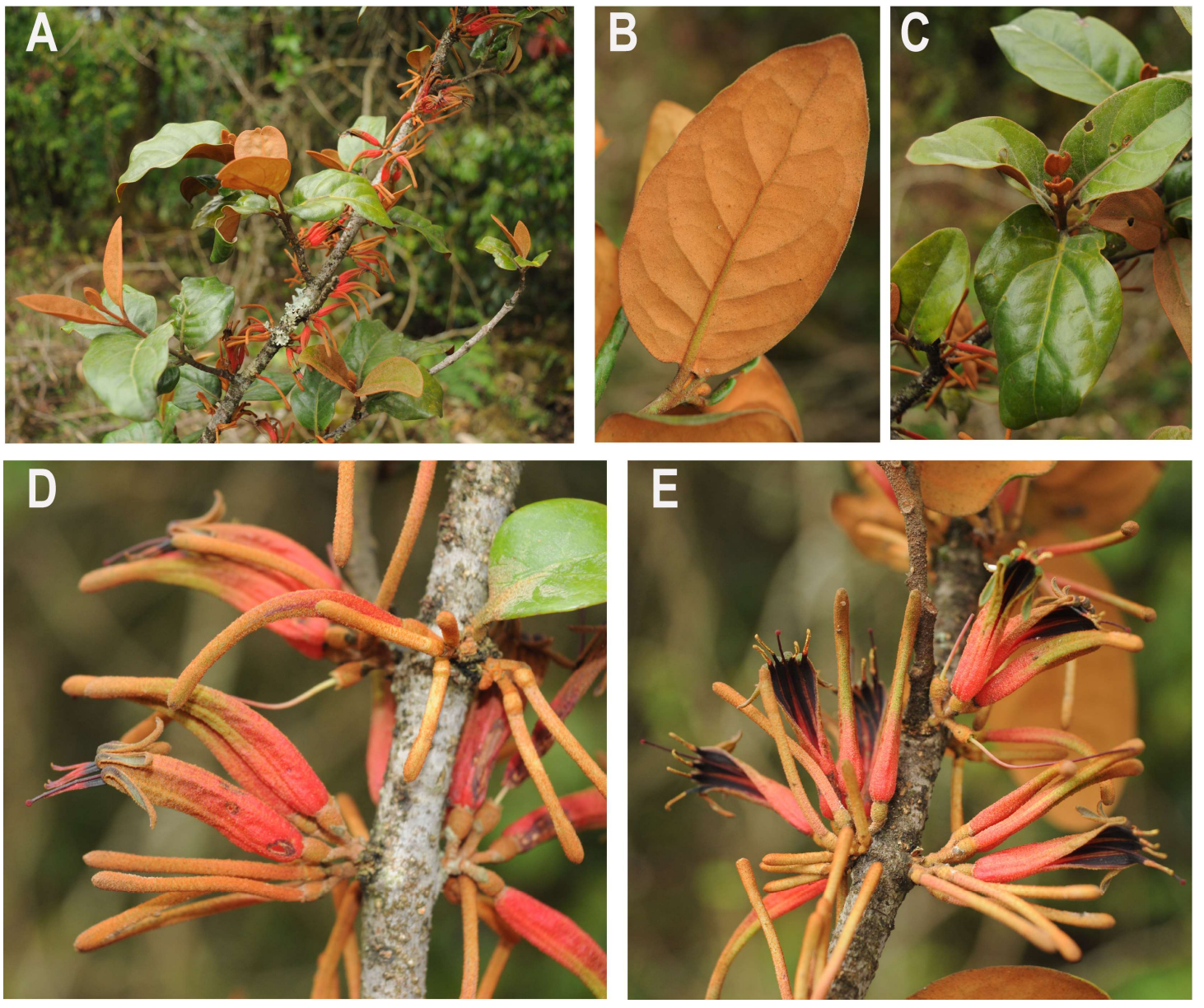

Figure 2. Morphology of Taxillus thibetensis. A: Habitat, Sapa, Lao Cai province, Vietnam. B: Abaxial and adaxial surfaces of leaf. C: Adaxial surfaces of leaf. D: Flower buds. E: Flowers.

of Taxillus thibetensis, so this species should be classified as Data Deficient (DD), according to IUCN Red List criteria (IUCN) [21]. Further field research may provide a more precise conservation assessment in the future.

Specimens examined: VIETNAM: Lao Cai prov. Sapa district, Ban Khoang commune, October 2019, Van Du Nguyen, Hung Manh Nguyen, Xuan Thanh Trinh \& Chi Toan Le DMTT35, DMTT36, DMTT54, DMTT55,
DMTT58, DMTT62 (HN). CHINA: Yunnan Prov., Jingping County, Fenshuiling, 13 April 2015, Bing Liu, Tianwen Xiao, Xiaoyang Yang $2894 \& 2906$ (PE).

\section{Acknowledgements}

We are grateful to Hung Manh Nguyen and Xuan Thanh Trinh for field assistance. This research is funded by Vietnam National 
Foundation for Science and Technology Development (NAFOSTED) under grant No. 106.03-2019.12.

\section{References}

[1] H. X. Kiu, M. G. Gilbert, Loranthaceae, Flora of China, Science Press \& Missouri Botanical Garden Press, Beijing \& St. Louis, 2003, pp. 220-239.

[2] D. L. Nickrent, V. Malécot, R. Vidal-Russell, J. P. Der, A Revised Classification of Santalales, Taxon, Vol. 592, 2010, pp. 538-558.

[3] B. Liu, C. T. Le, R. L. Barrett, D. L. Nickrent, Z. D. Chen, L. M. Lu, R. V. Russell, Diversification Agrees with Emergence of Tropical Forests and Radiation of Songbirds, Mol. Phylogenet. Evol., Vol. 124, 2018, pp. 199-212.

[4] H. H. Pham, An Illustrated Flora Vietnam, Young Publishing House, Ho Chi Minh City, 2003, pp. 951. (in Vietnamese)

[5] T. B. Nguyen, Loranthaceae, Checklist of Plant Species of Vietnam, Agricultural Publishing House, Hanoi, 2004, pp. 1182-1190. (in Vietnamese)

[6] N. H. Le, T. B. Tran, H. Q. Bui, V. H. Do, T. H. Bui, L. Averyanov, M. S. Nuraliev, Taxonomic Notes on Tolypanthus and Taxillus (Loranthaceae) in Vietnam, Including Lectotypifications and New National Records, Phytotaxa, Vol. 424, 2019, pp. 167-176.

[7] N. J. Turland, J. H. Wiersema, F. R. Barrie, W. Greuter, D. L. Hawksworth, P. S. Herendeen, S. Knapp, W. H. Kusber, D. Z. Li, K. Marhold, T. W. May, J. McNeill, A. M. Monro, J. Prado, M. J. Price, G.F. Smith, International Code of Nomenclature for algae, fungi, and plants (Shenzhen Code) adopted by the Nineteenth International Botanical Congress Shenzhen, China, July 2017. Regnum vegetabile 159. Koeltz Botanical Books, Glashütten Publishing House, 2018, pp. 254.

[8] R. V. Russell, D. L. Nickrent, Evolutionary Relationship in the Showy Mistletoe Family (Loranthaceae), Amer. J. Bot., Vol. 95, 2008, pp. 1015-1029.

[9] H.J. Su, J.M. Hu, F.E. Anderson, J.P. Der, D.L. Nickrent, Phylogenetic relationships of Santalales with insights into the origins of holoparasitic Balanophoraceae, Taxon 64 (2015) 491-506.

[10] C. T. Le, Phylogeny Biogeography and
Diversification of Santalales. Doctoral thesis. Institute of Botany, Chinese Academy of Science, Beijing, 2018.

[11] C. T. Le, V. D. Nguyen, T. X. Do, V. H. Nguyen, T. B. H. Pham, T. H. Phan, T. H. Nguyen, N. T. P. Hoang, Study on Morphology and Genetics of Scurrula chingii var. yunnanensis H. S. Kiu in C. Y. $\mathrm{Wu} \& \mathrm{H}$. W. Li. Proceeding of the $4^{\text {th }}$ national Scientific Conference on Biological Research and Teaching in Vietnam, 2020, pp. 447-453. (in Vietnamese) J. J. Doyle, J. L. Doyle, A Rapid DNA Isolation Procedure for Small Quantities of Fresh Leaf Tissue, Phytochem. Bull., Vol. 19, 1987, 1115.

[12] R. V. Russell, D. L. Nickrent, The First Mistletoes, Origins of Aerial Parasitism in Santalales, Mol. Phylogenet. Evol., Vol. 47, 2008, pp. 523-537.

[13] P. Taberlet, L. Gielly, G. Pautou, Universal Primers for Amplification of Three Non-coding Regions of Chloroplast DNA, Plant Mol. Biol., Vol. 17, 1991, pp. 1105-1109.

[14] M. Kearse, R. Moir, A. Wilson, S. S. Havas, M. Cheung, S. Sturrock, S. Buxton, A. Cooper, S. Markowitz, C. Duran, T. Thierer, B. Ashton, P. Mentjies, A. Drummond, Geneious Basic: an Integrated and Extendable Desktop Software Platform for the Organization and Analysis of Sequence Data, Bioinformatics, Vol. 28, 2012, pp. 1647-1649.

[15] Stamatakis, RAxML-VI-HPC Maximum Likelihood-based Phylogenetic Analyses with Thousands of Taxa and Mixed Models, Bioinformatics, Vol. 2221, 2006, pp. 2688-2690.

[16] Stamatakis, P. Hoover, J. Rougemont, A Rapid Bootstrap Algorithm for the RAxML Web Servers, Syst. Biol., Vol. 575, 2008, pp. 758-771.

[17] F. Ronquist, J. P. Huelsenbeck, MrBayes, Bayesian Phylogenetic Inference Under Mixed Modelsc Bioinformatics, Vol. 19, 2003, pp. 1572 -1574.

[18] D. Darriba, G. L. Taboada, R. Doallo, D. Posada, jModel Test 2: More Models, New Heuristics and Parallel Computing, Nature Methods, Vol. 9, 2012, pp. 772

[19] Rambaut, A. J. Drummond, Tracer, Version 1.4, 2007. http://beast.bio.ed.ac.uk/Tracer. (accessed on $13^{\text {rd }}$ February 2020.

[20] IUCN, IUCN Red List Categories and Criteria: Version 3.1. IUCN Species Survival Commission. IUCN, Gland, Switzerland and Cambridge, UK, 2001. 
Table S1. Voucher information and GenBank accession numbers for DNA sequences generated or used in this study. The sequences generated in this study begin with MZ. "-" indicates missing data.

\begin{tabular}{|c|c|c|c|c|c|c|c|}
\hline Species & Voucher/Source & $\begin{array}{l}\text { Country } \\
\text { of origin }\end{array}$ & LSU & SSU & $\begin{array}{l}\text { mat } \\
\mathrm{K}\end{array}$ & $r b c \mathbf{L}$ & $\begin{array}{l}\operatorname{trn} \mathrm{L}- \\
\mathrm{F} \\
\end{array}$ \\
\hline $\begin{array}{l}\text { Dendrophthoe longitub } \\
a \text { (Elmer) Danser }\end{array}$ & D. L. Nickrent 4010 (SIU) & Malaysia & $\begin{array}{l}\text { EU5 } \\
4436 \\
6\end{array}$ & - & $\begin{array}{l}\text { EU5 } \\
4442 \\
3\end{array}$ & - & $\begin{array}{l}\text { EU5 } \\
4448 \\
5\end{array}$ \\
\hline $\begin{array}{l}\text { Scurrula buddleioides } \\
\text { (Desr.) G. Don }\end{array}$ & Z. J. Qiu 0096 (PE) & China & $\begin{array}{l}\text { MG9 } \\
9940 \\
2\end{array}$ & $\begin{array}{l}\text { MG9 } \\
9947 \\
5\end{array}$ & $\begin{array}{l}\text { MG9 } \\
9942 \\
3\end{array}$ & $\begin{array}{l}\text { MG9 } \\
9945 \\
1\end{array}$ & $\begin{array}{l}\text { MG9 } \\
9949 \\
9\end{array}$ \\
\hline $\begin{array}{l}\text { Scurrula chingii (W.C. } \\
\text { Cheng) H.S. Kiu }\end{array}$ & B. Liu 1736 (PE) & China & $\begin{array}{l}\text { MG9 } \\
9940 \\
3 \\
\end{array}$ & $\begin{array}{l}\text { MG9 } \\
9947 \\
6 \\
\end{array}$ & $\begin{array}{l}\text { MG9 } \\
9942 \\
4 \\
\end{array}$ & $\begin{array}{l}\text { MG9 } \\
9945 \\
2 \\
\end{array}$ & $\begin{array}{l}\text { MG9 } \\
9950 \\
0 \\
\end{array}$ \\
\hline $\begin{array}{l}\text { Scurrula ferruginea (Ja } \\
\text { ck) Danser }\end{array}$ & D. L. Nickrent 4008 (SIU) & Malaysia & $\begin{array}{l}\text { EU5 } \\
4439 \\
5\end{array}$ & $\begin{array}{l}\text { EU5 } \\
4434 \\
3\end{array}$ & $\begin{array}{l}\text { EU5 } \\
4445 \\
1\end{array}$ & $\begin{array}{l}\text { KF11 } \\
4863\end{array}$ & $\begin{array}{l}\text { EU5 } \\
4450 \\
5\end{array}$ \\
\hline Scurrula parasitica $\mathrm{L}$. & $\begin{array}{l}\text { D. L. Nickrent } 4004 \text { (SIU); } T \text {. } \\
\text { Yang BZXHDGK0047 (PE) }\end{array}$ & $\begin{array}{l}\text { Malaysia } \\
\text {, China }\end{array}$ & $\begin{array}{l}\text { EU5 } \\
4439 \\
7\end{array}$ & $\begin{array}{l}\text { EU5 } \\
4434 \\
5\end{array}$ & $\begin{array}{l}\text { EU5 } \\
4445 \\
1\end{array}$ & $\begin{array}{l}\text { MG9 } \\
9945 \\
4\end{array}$ & $\begin{array}{l}\text { MG9 } \\
9950 \\
2\end{array}$ \\
\hline $\begin{array}{l}\text { Scurrula philippensis } \\
\text { (Cham. \& Schltdl.) G. } \\
\text { Don }\end{array}$ & $\begin{array}{l}\text { T. Yang BZXHDGK0054 } \\
\text { (PE) }\end{array}$ & China & $\begin{array}{l}\text { MG9 } \\
9940 \\
5 \\
\end{array}$ & $\begin{array}{l}\text { MG9 } \\
9947 \\
8 \\
\end{array}$ & $\begin{array}{l}\text { MG9 } \\
9942 \\
6\end{array}$ & $\begin{array}{l}\text { MG9 } \\
9945 \\
5 \\
\end{array}$ & $\begin{array}{l}\text { MG9 } \\
9950 \\
3 \\
\end{array}$ \\
\hline $\begin{array}{l}\text { Scurrula } \\
\text { pulverulenta (Wall.) G. } \\
\text { Don }\end{array}$ & M. Devkota 661 (KATH) & Nepal & $\begin{array}{l}\text { EU5 } \\
4439 \\
6 \\
\end{array}$ & $\begin{array}{l}\text { EU5 } \\
4434 \\
4\end{array}$ & $\begin{array}{l}\text { EU5 } \\
4445 \\
2\end{array}$ & - & - \\
\hline $\begin{array}{l}\text { Taxillus chinensis (DC.) } \\
\text { Danser }\end{array}$ & $\begin{array}{l}\text { D. L. Nickrent } 4032 \text { (SIU); Z. } \\
\text { D. Chen \& C. T. Le } 36 \text { (PE) }\end{array}$ & $\begin{array}{l}\text { Malaysia } \\
\text { Vietnam }\end{array}$ & $\begin{array}{l}\text { EU5 } \\
4440 \\
5\end{array}$ & $\begin{array}{l}\text { EU5 } \\
4435 \\
0\end{array}$ & $\begin{array}{l}\text { EU5 } \\
4446 \\
0\end{array}$ & $\begin{array}{l}\text { MG9 } \\
9945 \\
6 \\
\end{array}$ & $\begin{array}{l}\text { MG9 } \\
9950 \\
4 \\
\end{array}$ \\
\hline $\begin{array}{l}\text { Taxillus sutchuenensis } \\
\text { (Lecomte) Danser }\end{array}$ & Z. D. Chen 20010418 (PE) & China & $\begin{array}{l}\text { MG9 } \\
9940 \\
6\end{array}$ & $\begin{array}{l}\text { MG9 } \\
9947 \\
9\end{array}$ & $\begin{array}{l}\text { MG9 } \\
9942 \\
7\end{array}$ & $\begin{array}{l}\text { MG9 } \\
9945 \\
7 \\
\end{array}$ & $\begin{array}{l}\text { MG9 } \\
9950 \\
5 \\
\end{array}$ \\
\hline $\begin{array}{l}\text { Taxillus thibetensis } \\
\text { (Lecomte) Danser }\end{array}$ & C. T. Le et al. DMTT $35(\mathrm{HN})$ & Vietnam & $\begin{array}{l}\text { MZ4 } \\
2026 \\
3 \\
\end{array}$ & - & - & \begin{tabular}{|l|} 
MZ4 \\
2024 \\
4 \\
\end{tabular} & $\begin{array}{l}\text { MZ4 } \\
2025 \\
6 \\
\end{array}$ \\
\hline $\begin{array}{l}\text { Taxillus thibetensis } \\
\text { (Lecomte) Danser }\end{array}$ & C. T. Le et al. DMTT $36(\mathrm{HN})$ & Vietnam & $\begin{array}{l}\text { MZ4 } \\
2026 \\
4\end{array}$ & $\begin{array}{l}\text { MZ4 } \\
2025 \\
1\end{array}$ & $\begin{array}{l}\text { MZ4 } \\
2023 \\
8\end{array}$ & $\begin{array}{l}\text { MZ4 } \\
2024 \\
5\end{array}$ & $\begin{array}{l}\text { MZ4 } \\
2025 \\
7\end{array}$ \\
\hline $\begin{array}{l}\text { Taxillus thibetensis } \\
\text { (Lecomte) Danser }\end{array}$ & C. T. Le et al. DMTT $54(\mathrm{HN})$ & Vietnam & $\begin{array}{l}\text { MZ4 } \\
2026 \\
5\end{array}$ & - & $\begin{array}{l}\text { MZ4 } \\
2023 \\
9\end{array}$ & $\begin{array}{l}\text { MZ4 } \\
2024 \\
6 \\
\end{array}$ & $\begin{array}{l}\text { MZ4 } \\
2025 \\
8\end{array}$ \\
\hline $\begin{array}{l}\text { Taxillus thibetensis } \\
\text { (Lecomte) Danser }\end{array}$ & C. T. Le et al. DMTT $55(\mathrm{HN})$ & Vietnam & $\begin{array}{l}\text { MZ4 } \\
2026 \\
6\end{array}$ & $\begin{array}{l}\text { MZ4 } \\
2025 \\
2\end{array}$ & $\begin{array}{l}\text { MZ4 } \\
2024 \\
0\end{array}$ & $\begin{array}{l}\text { MZ4 } \\
2024 \\
7\end{array}$ & $\begin{array}{l}\text { MZ4 } \\
2025 \\
9 \\
\end{array}$ \\
\hline $\begin{array}{l}\text { Taxillus thibetensis } \\
\text { (Lecomte) Danser }\end{array}$ & C. T. Le et al. DMTT $58(\mathrm{HN})$ & Vietnam & $\begin{array}{l}\text { MZ4 } \\
2026 \\
7\end{array}$ & $\begin{array}{l}\text { MZ4 } \\
2025 \\
3\end{array}$ & $\begin{array}{l}\text { MZ4 } \\
2024 \\
1\end{array}$ & $\begin{array}{l}\text { MZ4 } \\
2024 \\
8\end{array}$ & $\begin{array}{l}\text { MZ4 } \\
2026 \\
0 \\
\end{array}$ \\
\hline $\begin{array}{l}\text { Taxillus thibetensis } \\
\text { (Lecomte) Danser }\end{array}$ & C. T. Le et al. DMTT $62(\mathrm{HN})$ & Vietnam & $\begin{array}{l}\text { MZ4 } \\
2026 \\
8\end{array}$ & $\begin{array}{l}\text { MZ4 } \\
2025 \\
4\end{array}$ & $\begin{array}{l}\text { MZ4 } \\
2024 \\
2\end{array}$ & $\begin{array}{l}\text { MZ4 } \\
2024 \\
9\end{array}$ & $\begin{array}{l}\text { MZ4 } \\
2026 \\
1\end{array}$ \\
\hline
\end{tabular}




\begin{tabular}{|c|c|c|c|c|c|c|c|}
\hline $\begin{array}{l}\text { Taxillus thibetensis } \\
\text { (Lecomte) Danser }\end{array}$ & B. Liu 2894 (PE) & China & $\begin{array}{l}\text { MG9 } \\
9940 \\
7\end{array}$ & $\begin{array}{l}\text { MG9 } \\
9948 \\
0\end{array}$ & $\begin{array}{l}\text { MG9 } \\
9942 \\
8 \\
\end{array}$ & $\begin{array}{l}\text { MG9 } \\
9945 \\
8 \\
\end{array}$ & $\begin{array}{l}\text { MG9 } \\
9950 \\
6 \\
\end{array}$ \\
\hline $\begin{array}{l}\text { Taxillus thibetensis } \\
\text { (Lecomte) Danser }\end{array}$ & B. Liu 2906 (PE) & China & $\begin{array}{l}\text { MZ4 } \\
2026 \\
9 \\
\end{array}$ & $\begin{array}{l}\text { MZ4 } \\
2025 \\
5 \\
\end{array}$ & $\begin{array}{l}\text { MZ4 } \\
2024 \\
3 \\
\end{array}$ & $\begin{array}{l}\text { MZ4 } \\
2025 \\
0 \\
\end{array}$ & $\begin{array}{l}\text { MZ4 } \\
2026 \\
2 \\
\end{array}$ \\
\hline Taxillus tsaii S.T. Chiu & Z. D. Chen 20110126 (PE) & China & $\begin{array}{l}\text { MG9 } \\
9940 \\
8\end{array}$ & $\begin{array}{l}\text { MG9 } \\
9948 \\
1\end{array}$ & $\begin{array}{l}\text { MG9 } \\
9942 \\
9\end{array}$ & $\begin{array}{l}\text { MG9 } \\
9945 \\
9\end{array}$ & $\begin{array}{l}\text { MG9 } \\
9950 \\
7\end{array}$ \\
\hline
\end{tabular}

